\title{
Atmospheric-turbulence-induced power-fade statistics for a multiaperture optical receiver
}

\author{
Aniceto Belmonte, A. Comerón, J. A. Rubio, J. Bará, and E. Fernández
}

\begin{abstract}
To estimate the probability distributions of power fades, we consider two basic types of disturbance in electromagnetic wave propagation through atmospheric turbulence: wave-front intensity fluctuations and wave-front distortion. We assess the reduction in the cumulative probability of losses caused by these two effects through spatial diversity by using a multiaperture receiver configuration. Degradations in receiver performance are determined with fractal techniques used to simulate the turbulenceinduced wave-front phase distortion, and a log normal model is assumed for the collected power fluctuations. (c) 1997 Optical Society of America

Key words: Atmospheric optics, multiaperture systems, averaging.
\end{abstract}

\section{Introduction}

This study is concerned with the estimation of the reduction in the cumulative probability of total losses induced by atmospheric turbulence in an optical detection system. In nonguided optical communication links contained totally (ground-ground, planeground, etc.) or partially (satellite-ground, satelliteplane, etc.) within the atmosphere, the effects of turbulence-induced intensity variation (scintillation) and distortion of an optical wave front (i.e., the isophase surface) on system performance are a higher bit-error rate, an increased probability of burst error, and difficulties in maintaining the tracking in the receiver station. Whereas wave-front intensity fluctuations ${ }^{1}$ translate into fluctuations of the power collected by the receiver aperture, the wave-front distortion, essentially a phase ripple superimposed on an average wave-front inclination with respect to the system optical axis, ${ }^{2}$ causes a deformation of the focal spot and a jitter of its position around the focus, which may drive part of the power off the surface of the focal-plane collecting element. Both intensity fluctuation and focal-spot deformation and jitter re-

A. Belmonte, A. Comerón, J. A. Rubio, and J. Bará are with the Department of Signal Theory and Communications, Universitat Politècnica de Catalunya, UPC, 08034 Barcelona, Spain. E. Fernández is with the Department of Software and Communications, Computadores y Redes Informáticas, S.A., 28760 Madrid, Spain.

Received 30 October 1996; revised manuscript received 11 March 1997.

0003-6935/97/338632-07\$10.00/0

(C) 1997 Optical Society of America sult in fades of the signal power reaching the detector and give rise to the performance impairments mentioned above.

Here we present techniques developed to estimate in a complete and realistic fashion the reduction in the cumulative probability of total losses induced by the two effects described above through spatial diversity obtained with multiaperture architectures in the receiver configurations. Some early studies dealing with multiaperture receivers are described in Refs. 3 and 4. In this way we can achieve enhanced averaging effects through the decorrelation of the disturbances on the wave-front portions arriving at different apertures enhanced with respect to those effects obtained by employing a larger single aperture. In section 2 we present a generalization to a multiaperture system of the averaging factor necessary to derive the collected power variance from the intensity spatial covariance function. ${ }^{5}$ In Section 3 fractal techniques are used to generate simulations of instantaneous turbulence-distorted wave fronts and their corresponding instantaneous point-spread function. ${ }^{6}$ In Section 4 both collected power fluctuations and wave-front distortion effects, assumed independent, are combined into a single function, giving the probability that a given fade of the power reaching the receiver system is exceeded.

\section{Losses Induced by Fluctuations of the Collected Power}

When a large aperture is used to collect light propagated through the atmosphere, it performs an averaging operation on the irradiance fluctuations, resulting in collected power fluctuations that are not 


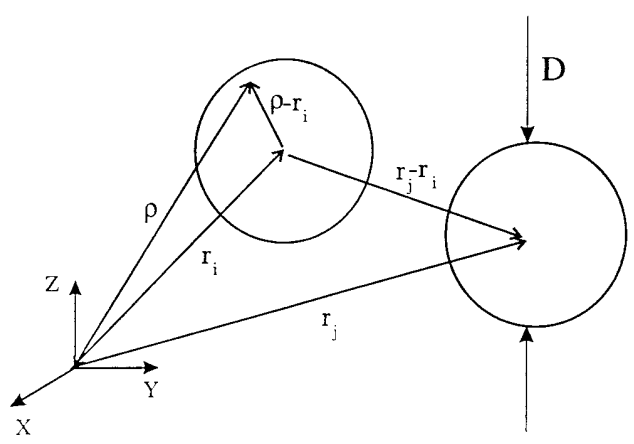

Fig. 1. Geometry of circular apertures with a center-to-center separation $\left|\mathbf{r}_{i}-\mathbf{r}_{j}\right|$ and an individual aperture diameter $D$.

as large as those that would be obtained from a point receiver. However, although the impact of these effects could be reduced to some extent by an increase in the receiving aperture size, this might turn out to be an expensive solution. Alternatively, one can obtain the spatial diversity implied by a large aperture by instead employing several smaller apertures that can be set at large distances from each other to achieve an enhanced averaging effect through decorrelation of the disturbances on the wave-front portions arriving at each aperture. Thus to estimate the statistics of power fades resulting from fluctuations of the collected power arising from wave-front intensity fluctuations, one would consider a generalization of the averaging factor used to derive fluctuations of the collected power from the spatial covariance function of intensity fluctuations. ${ }^{5}$

\section{A. Received Power Fluctuations}

We assume that the basic configuration of the optical receiver consists of $N$ identical apertures, each one characterized by an intensity pupil function $W\left(\mathbf{r}_{i}\right)$, where $\mathbf{r}_{i}$ is the position of the $i$ th aperture center (Fig. 1). Then the instantaneous power collected by the multiaperture system is

$$
P=\sum_{i=1}^{N} \int_{S_{i}} W\left(\rho_{i}-\mathbf{r}_{i}\right) I\left(\rho_{i}\right) \mathrm{d} \rho_{i},
$$

where $I\left(\rho_{i}\right)$ is the wave intensity over the $i$ th aperture, and under the assumption of identical subapertures its variance $\sigma_{p}{ }^{2}=\left\langle(P-\langle P\rangle)^{2}\right\rangle$ can be written $\operatorname{as}^{5}$

$$
\sigma_{P}^{2}=\sum_{i=1}^{N} \sum_{j=1}^{N} C_{P}\left(\mathbf{i}-\mathbf{r}_{j}\right)
$$

Here $C_{P}(\rho)$ is the spatial covariance function of the power collected by an aperture calculated as the correlation of the spatial impulse-response function of an aperture $K_{W}(\rho)$ with the spatial covariance function of intensity fluctuations $C_{I}(\rho)$ :

$$
C_{P}\left(\rho=\int_{S^{\prime}} K_{W}\left(\rho^{\prime}\right) C_{I}\left(\rho^{\prime}+\rho\right) \mathrm{d} \rho^{\prime} .\right.
$$

Equation (2) can be used to compute the variance of the power collected by a multiaperture system for any number of identical subapertures with given relative positions once the spatial covariance function of intensity fluctuations $C_{I}(\rho)$ is known.

As an example of the previous results, let us consider the most simple configuration for the collected system: a single aperture. From Eqs. (2) and (3),

$$
\sigma_{P}^{2}=C_{P}(0)=\int_{S} K_{W}(\rho) C_{I}(\rho) \mathrm{d} \rho,
$$

we obtain Fried's classical expression ${ }^{7}$ that describes how the collected power fluctuations decrease when the single collecting aperture size increases. Although it is clear from Eq. (4) that the relationship between the variance $\sigma_{P}{ }^{2}$ and the aperture is not straightforward, for a large aperture of diameter $D$, and under the assumption that Tatarski's theory of wave propagation in a turbulent medium ${ }^{8}$ applies correctly, Dainty et al. ${ }^{9}$ showed that Eq. (4) yields the known $D^{-7 / 3}$ law for scintillation variance. ${ }^{10}$

\section{B. Computation of the Probability of Losses}

In this section we examine how the power fadings caused by turbulence-induced power fluctuations degrade the reliability of optical link performance. It is useful to define the log power $\chi_{P}$ as

$$
\chi_{P}=\ln \left(P / P_{0}\right),
$$

where $P_{0}=\langle P\rangle$ is the mean power. Tatarski's theory $^{8}$ predicts that, in the absence of aperture averaging, the statistics of this magnitude are approximately governed by the normal law,

$$
p_{\chi_{P}}\left(\chi_{P}\right)=\frac{1}{\left(2 \pi \sigma_{\chi_{P}}{ }^{2}\right)^{1 / 2}} \exp \left[-\frac{\left(\chi_{P}-\chi_{P 0}\right)^{2}}{2 \sigma_{\chi P}}\right],
$$

where $\chi_{P 0}$ and $\sigma_{\chi P}{ }^{2}$ are the mean and the variance of log power, respectively. (Although observations have shown small deviations from this law, ${ }^{9,11}$ when we consider the effect of averaging by a large collected power aperture, these deviations are negligible ${ }^{12}$ and do not affect our calculations.) In that case it follows that the distribution function of the power fluctuations is $\log$ normal and is given by ${ }^{13}$

$$
p_{P}(P)=\frac{1}{\left(2 \pi \sigma_{\chi_{P}}\right)^{1 / 2}} \frac{1}{2 P} \exp \left\{-\frac{1}{2 \sigma_{\chi_{P}}}\left[\frac{1}{2} \ln \left(\frac{P}{P_{0}}\right)+\sigma_{\chi_{P}}{ }^{2}\right]\right\},
$$

where, under the log normality hypothesis, $\chi_{P 0}=$ $-(1 / 2) \sigma_{\chi P}{ }^{2}$. In Eq. (7) the probability distribution for power is characterized by the variance of log power. However, under the log normality hypothesis one can relate the variance of the collected power, the most common measure of the magnitude of power fluctuations, to the log power variance through

$$
\sigma_{P}^{2}=P_{0}^{2}\left[\exp \left(\sigma_{\chi_{p}}^{2}\right)-1\right]
$$




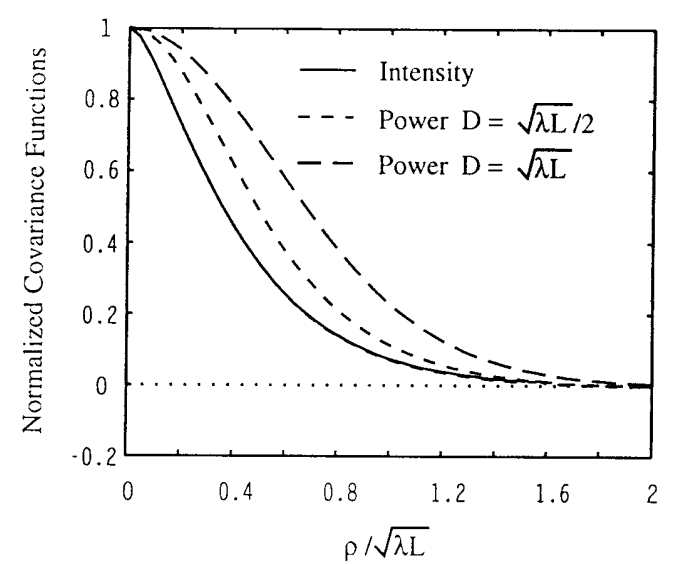

Fig. 2. Intensity normalized covariance function versus normalized spatial separation for spherical wave-propagation geometry. The corresponding collected power covariance is shown for two aperture diameters $D . \quad \lambda$ is the wavelength and $L$ is the propagation path length, yielding a $\sqrt{\lambda L}$ intensity correlation length when, as in this case, the covariance function is computed from Rytov's approximation.

It is usual to express the relation between a given power level $P$ and the corresponding powerfluctuation-induced loss $L_{P}$ in decibels as

$$
L_{P}=-10 \log \left(P / P_{0}\right)
$$

The probability of power-fluctuation-induced losses is obtained from Eqs. (7) and (9). $\sigma_{\chi P}{ }^{2}$ is calculated through Eq. (8) and the expression for the variance of the collected power ${\sigma_{P}}^{2}$ [Eq. (2)] in a multiaperture receiving system.

As an example of application of the previous results, let us consider several simple configurations for the receiver system, allowing us to analyze the evolution of the probability that a given powerfluctuation-induced loss level is exceeded versus some significant parameters. We use the intensity covariance function $C_{I}(\rho)$ computed under the hypothesis of spherical wave geometry and turbulence conditions yielding $C_{I}(0)={\sigma_{I}}^{2}=0.1$. That makes the intensity covariance approximately proportional to the log-amplitude covariance, which can be computed with Rytov's approximation. ${ }^{1}$ Figure 2 shows the intensity normalized covariance function versus the normalized spatial separation and the corresponding collected power covariance [Eq. (3)] for different aperture diameters.

Figure 3 shows the probability that a given loss level is exceeded versus the number of subapertures. For this we consider several numbers of subapertures arranged in a row. In this way it is possible to study the effect of the number of subapertures. Figure 4 gives the probability that a given power-fluctuationinduced loss level is exceeded for a two-aperture array and a square-geometry array of $2 \times 2$ subapertures versus the distance between contiguous subapertures. As expected, the results of the calculations show that an increase in the number of sub-

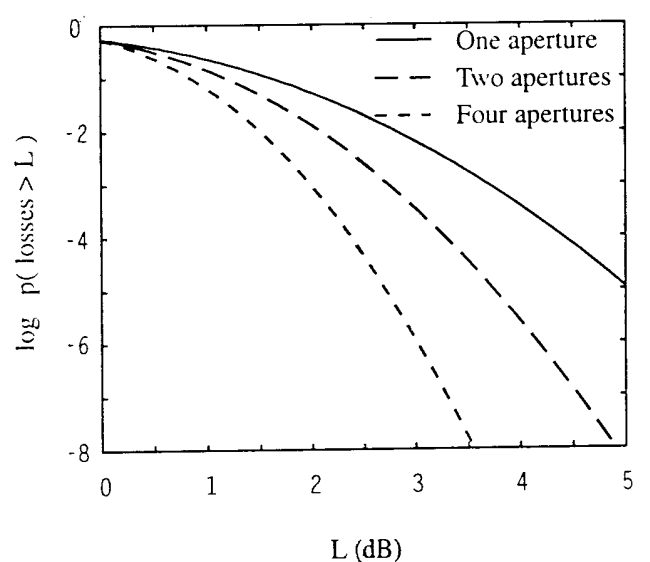

Fig. 3. Probability that the power-fluctuation-induced loss level on the abscissa is exceeded as a function of the number of subapertures arranged in a row. The atmospheric path is characterized by the intensity variance of ${\sigma_{I}}^{2}=0.1$ and the normalized intensity covariance function shown in Fig. 2. $\quad D=\sqrt{\lambda L} / 2$ and $l=\sqrt{\lambda L}$, where $D$ is the individual aperture diameter and $l$ is the centerto-center distance between contiguous subapertures.

apertures or the separation distance causes a decrease in the estimated power-fade probabilities.

\section{Losses Induced by Point-Spread Function Distortion}

In an aberration-free optical system the point-spread function is described by the Airy function. However, atmospheric turbulence distorts the wave front, so that the Airy function no longer correctly describes the short-exposure focal spot. In fact, we have a distorted instantaneous spot with its centroid displaced from the system focus owing to the average wave-front tilt at the system input. We use a simple model to describe the focal spot, where we approximate the effect of the turbulence-induced distortion of the point-spread function by considering a longexposure Gaussian point-spread function, which results from an instantaneous Gaussian focal spot with a centroid displacement from the focus following independent Gaussian laws with the same variance along two perpendicular axes. ${ }^{6}$ This allows us to use a semianalytical procedure to compute the losses occurring because the focal spot is driven off the focalplane power-collecting area. However, in addition to the spot motion, when a wave travels through the atmosphere and is focused by an optical system, the turbulence-induced wave-front distortion gives rise to other deformations of the system point-spread function. It has been shown ${ }^{6}$ that the hypothesis of a Gaussian instantaneous spot may lead to a serious underestimation of the probability that a given loss level is exceeded owing to turbulence-induced distortion of the point-spread function, at least for relatively high-turbulence conditions. So we propose to use a more realistic model that takes into account the full complexity of the wave front-and hence the focal-spot distortion-through fractal techniques, allowing us to simulate the received wave front numerically with more computing efficiency than other 
(a)

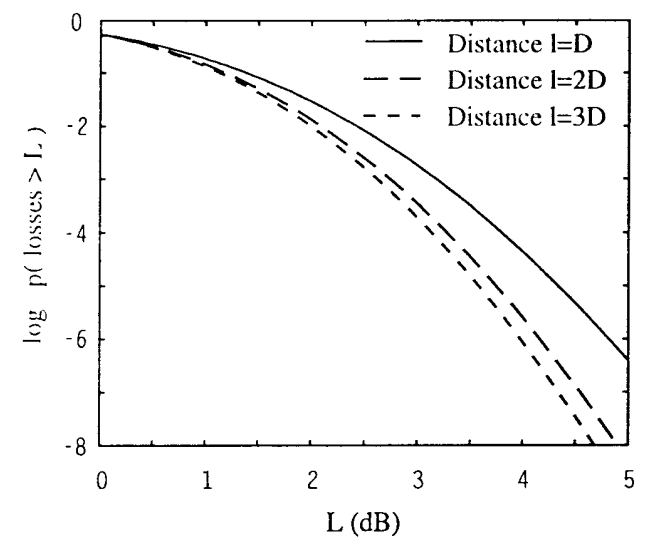

(b)

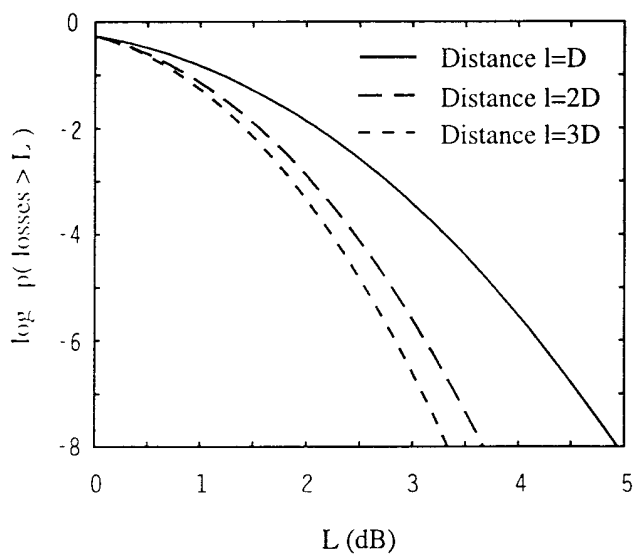

Fig. 4. Probability that a given power-fluctuation-induced loss level on the abscissa is exceeded for (a) a two-aperture array and (b) a square array of $2 \times 2$ subapertures. The same turbulence assumptions as in Fig. 3 are made for the atmospheric path, $D=$ $\sqrt{\lambda L} / 2$. Calculations are shown as a function of the distance $l$ between the subapertures.

methods (e.g., spectral techniques, ${ }^{14}$ Zernike polynomials $\left.{ }^{15}\right)$.

\section{A. Fractal Nature of the Wave Fronts}

Recently, wave fronts having traveled through the turbulent atmosphere have been identified as fractal surfaces. ${ }^{16,17}$ By using the Kolmogorov assumption concerning the power spectrum of index-of-refraction fluctuations in the atmosphere in the so-called inertial range, we can describe the distorted wave front by the phase-structure function ${ }^{2}$

$$
D_{S}(\rho)=6.88\left(\rho / r_{0}\right)^{5 / 3},
$$

where $r_{0}$ is the well-known Fried's coherence diameter. Knowing the relationship between the structure function and the power spectrum of the wavefront phase,

$$
D_{S}(\rho)=2 \int_{0}^{\infty} \Phi_{S}(\mathbf{K})[1-\exp (j \mathbf{K} \cdot \rho)] \mathrm{d} \mathbf{K}
$$

we can show that, like $D_{S}(\rho), \Phi_{S}(\mathbf{K})$ also has a powerlaw dependence ${ }^{18}$ :

$$
\Phi_{S}(\mathbf{K})=0.023 r_{0}^{-5 / 3} K^{-11 / 3} .
$$

On the other hand, we can consider the fractal surfaces from the fractional Brownian motion family ${ }^{19}$ (an extension of the classical Brownian motion), with a process $B$ of this family being characterized by a structure function with a power-law dependence of

$$
D_{B}(\rho) \propto \rho^{2 H}
$$

and a power spectrum given by

$$
\Phi_{B}(K) \propto K^{-(2 H+E)},
$$

where $H$ is the Hurst parameter and $E$ is the standard topological dimension of the generalized surface. The difference between both parameters describes the ruggedness of the surface through the relation

$$
F=E-H+1,
$$

where $F$ is interpreted as the fractal dimension of the surface. Thus if, as some authors ${ }^{16}$ have done, we compare the previous phase-structure function and power spectrum of Eqs. (10) and (12) with relations (13) and (14), we can identify the wave front, where $E=2$, as a fractional Brownian motion with a Hurst parameter of $H=5 / 6$ and a fractal dimension of $F=$ $13 / 6$.

\section{B. Computation of the Probability of Losses}

Since wave fronts have been identified as fractal surfaces, it is possible to use algorithms developed for modeling fractal surfaces in computer graphic problems. An efficient algorithm for the generation of fractals is an improved version of the midpoint displacement algorithm. ${ }^{17}$ The algorithm is based on building a grid with the required phase-structure function: The four starting samples, generated according to the structure function [Eq. (10)], are used to produce a central sample by linear interpolation and the addition of a random displacement; procedures of interpolation and displacement in two dimensions, such as that previously described, are repeated until the grid has the desired number of samples.

Given the simulated instantaneous wave front $S(u$, $v$ ) over the receiver plane $(u, v)$, we can compute the instantaneous spot $I_{S}(x, y)$ over the focal plane $(x, y)$ with a simple Fourier transform operation,

$$
I_{S}(x, y)=1 /(f \lambda)^{2}\left|F_{x / f \lambda, y f \lambda}\{W(u, v) \exp [j S(u, v)]\}\right|^{2},
$$

where $f$ is the receiving optical system focal length, $\lambda$ is the radiation wavelength, and $W(u, v)$ is the lens pupil function used to focus the wave (see Fig. 5).

Therefore to find the loss level caused by overflow of the active area is a conceptually easy task: We simply integrate the simulated point-spread function 


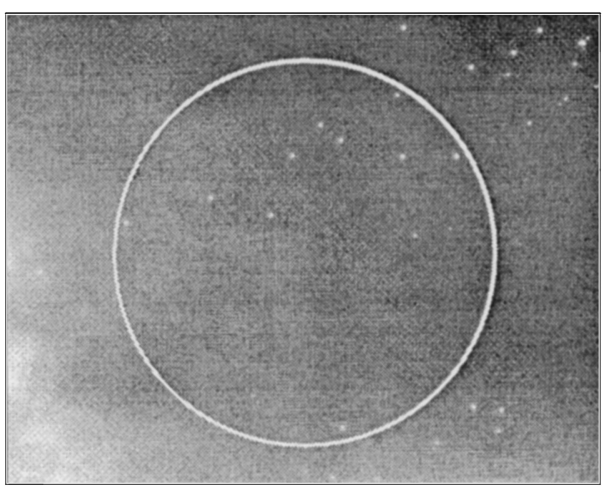

Wave front (Aperture)

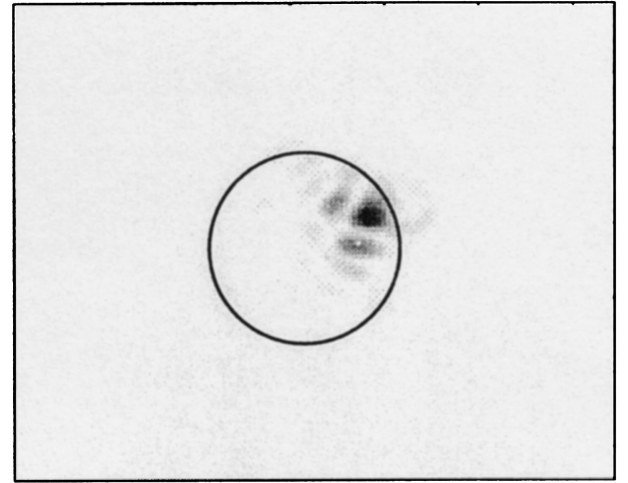

Point-spread function (Collecting area)

Fig. 5. Example of a fractal wave front generated by an improved version of the midpoint displacement algorithm. Given the simulated instantaneous wave front, we can compute the instantaneous point-spread function using a simple Fourier transform.

$I_{S}(x, y)$ over the focal-plane collecting area $\pi R_{D}{ }^{2}$ placed on the focus of the collecting lens. In decibels,

$$
L_{S}=-10 \log \left[1-\iint_{x^{2}+y^{2} \leq R_{D}{ }^{2}} I_{S}(x, y) \mathrm{d} x \mathrm{~d} y\right] .
$$

In the general case, in which we assume again that the configuration of the optical receiver system consists of $N$ identical apertures with the powercollecting elements on their foci, the apertures are placed on the instantaneous simulated wave front $S(u, v)$. The pupil function $W_{i}(u, v)$ corresponding to the $i$ th aperture can be used with Eqs. (16) and (17) to evaluate the $i$ th point-spread function $I_{S i}(x, y)$ and the loss $L_{S i}$ caused by overflow of the $i$ th detector. The repetition of the process of wave-front simulation, computation of point-spread functions, and evaluation of losses $\sum_{i=1}{ }^{N} L_{S i}$ allows us to estimate the probability law of losses.

Figures 6 and 7 show the probability that a given loss level is exceeded for the same sets of identical apertures considered in Subsection 2.B (Figs. 3 and 4) for the receiver system. These simple configurations allowed us to analyze the evolution of the statistics power fade induced by point-spread function distortion versus the number of subapertures and the distance between them. We carried out computations assuming a $10^{-4}$-rad field of view (which could correspond to a $100-\mu \mathrm{m}$ diameter detector with a $1-\mathrm{m}$ focal length) and turbulence conditions yielding $r_{0} /$ $D=0.1$. (For 25-cm individual aperture diameters, the corresponding Fried's coherence diameter $r_{0}$ would be $2.5 \mathrm{~cm}$; although this is a typical value for diurnal optical atmospheric links, for nighttime links it could be a pessimistic assumption.) As should be expected, the probability that a loss level is exceeded decreased when we considered an increase of both the number of subapertures and the separation distance between them.

\section{Computation of the Probability of Total Losses}

To account for the combined effect of losses induced by point-spread function distortion and intensity scintillation, we recognize that the total logarithmic loss $L$ is the sum of $L_{s}$ and $L_{P}$. It is then easily shown that, if $L_{s}$ and $L_{P}$ are assumed independent (i.e., point-spread function or phase distortion and intensity scintillation are independent ${ }^{20}$ ), the probability distribution of the total losses in decibels can be computed by convolution of the probability density function of the power-fluctuation losses with the probability density function of losses caused by pointspread function deformation.

Figure 8 shows the probability that a given loss level is exceeded owing to the combined effect of scintillation and spot distortion. We considered an array of two subapertures separated by $(\sqrt{\lambda L})$ and a square-geometry $2 \times 2$ array of subapertures with $l=$

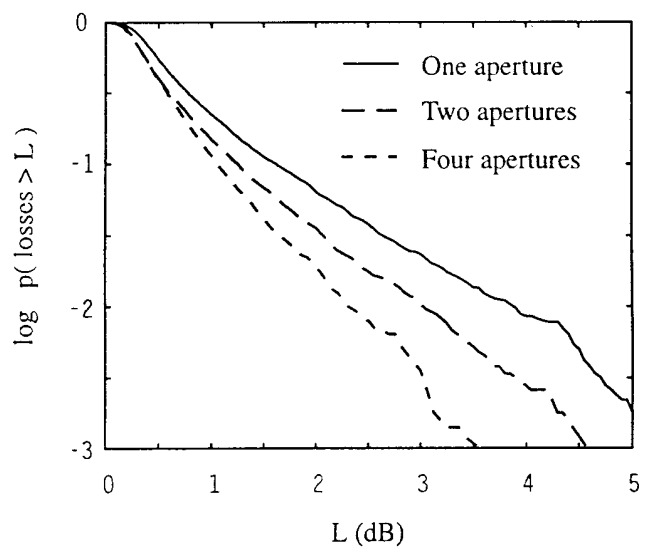

Fig. 6. Probability that the loss on the abscissa is exceeded owing to point-spread function distortion for several numbers of subapertures. Turbulence conditions yielding $r_{0} / D=0.1$ and individual apertures characterized by a $10^{-4}$-rad field of view and a diameter of $D=\sqrt{\lambda L} / 2$, with a center-to-center distance between them of $l=\sqrt{\lambda L}$, are assumed. 
(a)

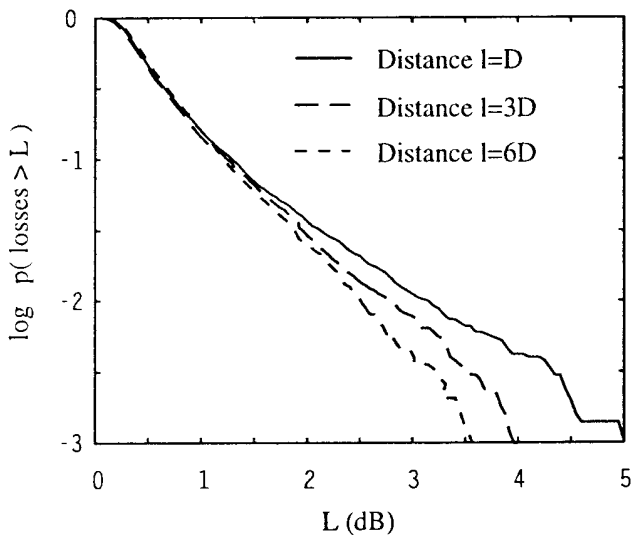

(b)

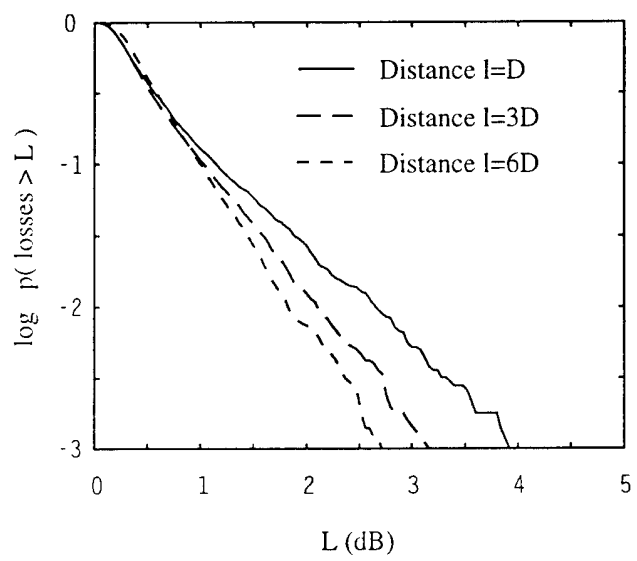

Fig. 7. Probability that a given spot-distortion-induced loss level on the abscissa is exceeded for (a) a two-aperture array and (b) a $2 \times 2$ square array of apertures. The same assumptions as in Fig. 6 are made for the atmospheric turbulence and the individual apertures. Calculations are shown for several distances $l$ between the apertures.

$\sqrt{\lambda L}$ sides and a $10^{-4}$-rad field of view for comparing the results with the probability of total losses for a single aperture with an area equivalent to one, four, or eight times the total array area. We carried out computations assuming turbulence conditions along the atmospheric path yielding $r_{0} / D=0.1$, an intensity variance of ${\sigma_{I}}^{2}=0.1$, and the normalized intensity covariance function shown in Fig. 2. In any case, it seems clear that the multiaperture configuration represents a more effective option to provide an enhanced performance of an optical receiver system. As can be seen, with a single aperture we obtained larger probabilities of total loss level even when the corresponding area was larger than the total array area.

In general, for arbitrary turbulence conditions and multiaperture configuration, it is not possible to tell $a$ priori which effect among scintillation and point-spread function distortion contributes the most to the total loss resulting from convolution. Rather, it is necessary to consider both particular atmospheric-turbulence conditions (through the (a)

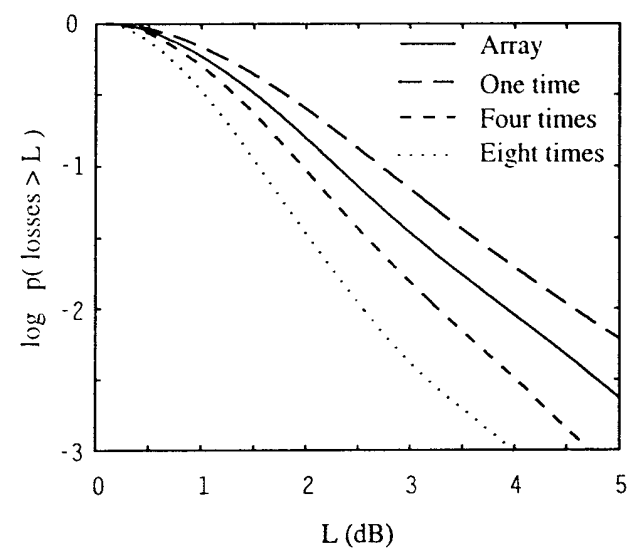

(b)

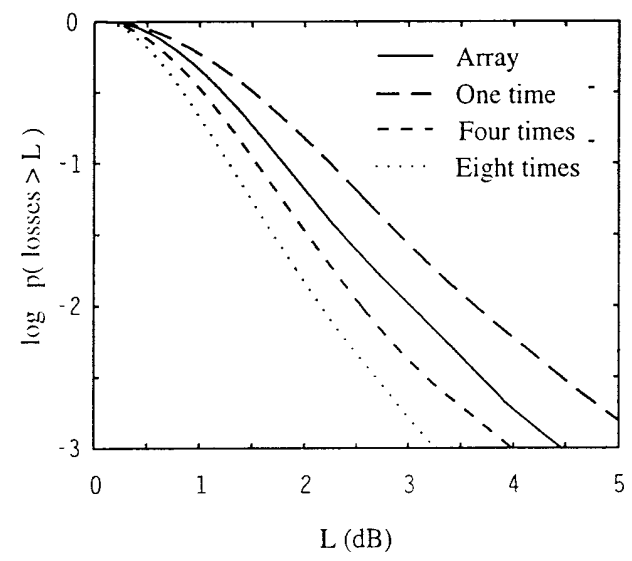

Fig. 8. Probability that the loss on the abscissa is exceeded owing to the combined effect of scintillation and focal-spot distortion: (a) a two-subaperture array with the center-to-center separation of $l=$ $\sqrt{\lambda L}$ and (b) a $2 \times 2$ square array of subapertures lying on the vertices of a square of $l=\sqrt{\lambda L}$ each side. In both cases individual subapertures characterized by a $10^{-4}$-rad field of view, a diameter of $D=\sqrt{\lambda L} / 2$, and turbulence conditions yielding $r_{0} / D=0.1$, an intensity variance of $\sigma_{I}^{2}=0.1$, and the normalized intensity covariance function shown in Fig. 2 are assumed. The results are compared with the probability of total losses for a single subaperture with an area equivalent to one, four, or eight times the total array area.

variance of the intensity ${\sigma_{I}}^{2}$ and the Fried's coherence diameter $r_{0}$ ) and multiaperture optical receiver designs (i.e., diameters and architecture of the multiple apertures). So, regarding the examples in the figures, scintillation and point-spread function distortion contributions are quite similar. However, turbulence conditions yielding lower $\sigma_{I}{ }^{2}$ and $r_{0}$ values (for example, a nonuniform path with high atmospheric turbulence near the receiver and weak turbulence far from it) could produce a distorted point-spread function preponderant in the loss process. On the other hand, paths with high turbulence near the transmitter could lead to situations in which scintillation would be the most important contribution in the computation of the probability of total loss. 


\section{Conclusions}

Losses produced by turbulence-induced intensity fluctuations and focal-spot deformation can cause severe impairments in the performance of atmospheric optical communication systems. We have studied the effect of reduction of the probability of losses in atmospheric optical link receivers through optical averaging by using multiaperture configurations. On the one hand, the key to the analysis was the development of fractal techniques to deal with the full complexity of focal-spot deformation and quivering about the focus position; on the other hand, we took into account the contribution of collected power fades through the spatial covariance function of the intensity fluctuations and a formulation that generalizes the single-aperture averaging effect to a multipleaperture receiver. Under the assumption that losses caused by turbulence-induced fluctuations of the collected power are independent of losses produced by the point-spread function distortion, we computed the probability distribution of the total logarithmic losses by convolving the probability density of the power-fluctuation losses with the probability density function of the losses caused by point-spread function deformation.

As seen in the study, it is tempting to conjecture that, with respect to atmospheric effects, a multiaperture receiver with small apertures may be a lowcost option providing performances similar to systems based on larger and more expensive single apertures.

This study has been carried out under European Space Agency contract 8973/90/NL/SG and purchase order ESP 114918, as well as Spain's Comisión Interministerial de Ciencia Y Tecnología grant ESP 978/90.

\section{References}

1. R. E. Hufnagel, "Propagation through atmospheric turbulence," in Infrared Handbook (Office of Naval Research, Department of the Navy, Arlington, Va., 1978).

2. D. L. Fried, "Statistics of a geometric representation of wavefront distortion," J. Opt. Soc. Am. 55, 1427-1435 (1965).

3. S. Rosenberg and M. C. Teich, "Photocounting array receivers for optical communication through the lognormal atmospheric channel. 2: Optimum and suboptimum receiver performance for binary signaling," Appl. Opt. 12, 2625-2635 (1973).

4. J. H. Churnside and M. C. McIntyre, "Averaged threshold receiver for direct detection of optical communications through the lognormal atmospheric channel," Appl. Opt. 16, 2669-2676 (1977).

5. A. Belmonte, A. Comerón, J. Bará, and J. A. Rubio, “Averaging of collected-power fluctuations by a multiaperture receiver system," Opt. Eng. 35, 2775-2778 (1996).

6. A. Belmonte, A. Comerón, J. Bará, J. A. Rubio, E. Fernández, and P. Menéndez-Valdés, "The impact of the point-spread function on the performance of a multiple-aperture optical ground station," in Atmospheric Propagation and Remote Sensing IV, J. C. Dainty, ed. Proc. SPIE 2471, 324-334 (1995).

7. D. L. Fried, "Aperture averaging of scintillation," J. Opt. Soc. Am. 57, 169-175 (1967).

8. V. I. Tatarski, Wave Propagation in a Turbulent Medium (Dover, New York, 1967).

9. J. C. Dainty, B. M. Levine, B. K. Brames, and K. A. O’Donnell, "Measurements of the wavelength dependence and other properties of stellar scintillation at Mauna Kea, Hawaii," Appl. Opt. 21, 127-145 (1978).

10. S. H. Reiger, "Starlight scintillations and atmospheric turbulence," Astron. J. 68, 395-406 (1963).

11. E. Jakeman, G. Parry, E. R. Pike, and P. N. Pusey, "The twinkling of stars," Contemp. Phys. 19, 127-145 (1978).

12. A. Belmonte, "Effects of the atmospheric turbulence on the propagation of electromagnetic waves," (in Spanish), Ph.D. dissertation (Polytechnic University of Catalonia, Barcelona, Spain, 1995).

13. H. T. Yura and W. G. McKinley, "Optical scintillation statistics for IR ground-to-space laser communication systems," Appl. Opt. 22, 3353-3358 (1983).

14. B. L. McGlamery, "Computer simulation studies of compensation of turbulence degraded images," in Image Processing ( $\mathrm{Pa}$ cific Grove), J. C. Urbach, ed., Proc. SPIE 74, 225-233 (1976).

15. N. Roddier, "Atmospheric wavefront simulation using Zernike polynomials," Opt. Eng. 29, 1174-1180 (1990).

16. C. Schwartz, G. Baum, and E. N. Ribak, "Turbulent-degraded wave fronts as fractal surfaces," J. Opt. Soc. Am. A 11, 444451 (1994).

17. R. G. Lane, A. Glindemann, and J. C. Dainty, "Simulation of a Kolmogorov phase screen,” Waves Random Media 2, 209-224 (1992).

18. R. J. Noll, "Zernike polynomials and atmosphere turbulence," J. Opt. Soc. Am. 66, 207-211 (1976).

19. H. O. Peitgen and D. Saupe, eds., The Science of Fractal Images (Springer-Verlag, New York, 1988).

20. J. W. Goodman, Statistical Optics (Wiley, New York, 1985). 